\title{
PANORAMA INTERNACIONAL DO REPROCESSAMENTO DE PRODUTOS MÉDICOS DE USO ÚNICO
}

\author{
International overview of single-use medical devices reprocessing \\ Panorama internacional do reprocesamiento de productos médicos de uso único \\ Eliana Auxiliadora Magahães Costa'
}

RESUMO: Introdução: O reuso de produtos de uso único é uma realidade mundial e implica em grandes problemas. Objetivo: Analisar o sistema regulatório de reprocessamento de produtos médicos de uso único a nível internacional, incluindo o brasileiro. Método: Revisão narrativa da literatura, utilizando bases de dados com descritores específicos. Resultado: Internacionalmente, as políticas de reuso de produtos médicos de uso único tendem a prevenção de danos. As regulamentações variam desde protocolos bem estruturados, como o norte-americano, o australiano e o alemão, à ausência de normatização a nível nacional, como identificado em países desenvolvidos como Canadá, Japão e alguns países da União Europeia. Conclusão: Os controles regulatórios existentes apresentam lacunas que dificultam sua implementação tanto para os serviços de saúde quanto para os fabricantes. Uma metodologia alternativa seria a de um sistema regulatório de produtos de uso único centrado no controle dos processos em lugar dos atuais focados no controle do produto. Palavras-chave: Segurança do paciente. Reutilização de equipamento. Política de saúde.

ABSTRACT: Introduction: The reuse of single-use products occurs worldwide and it leads to major issues. Objective: To analyze the international regulatory framework for the reprocessing of single-use medical products, including the Brazilian regulations. Methods: This is a narrative review of the literature, using databases with specific descriptors. Results: Internationally, there are a variety of regulations on the reuse of single-use medical products that aim at preventing damage. The regulatory environment comprises well-structured protocols, such as the American, Australian, and German protocols, to lack of regulations at a national level, as identified in developed countries such as Canada, Japan, and some European countries. Conclusion: Current regulatory controls have considerable gaps that hinder their implementation by the health services and manufacturers. An alternative approach may be the formulation of a regulatory framework of single-use products focused on the control of the processes instead of the current control of products. Keywords: Patient safety. Equipment reuse. Health policy.

RESUMEN: Introducción: El reúso de productos de uso único es una realidad mundial e implica en grandes problemas. Objetivo: Analizar el sistema regulatorio de reprocesamiento de productos médicos de uso único a nivel internacional, incluyendo el brasileño. Método: Revisión narrativa de la literatura, utilizando bases de datos con descriptores específicos. Resultado: Internacionalmente, las políticas de reúso de productos médicos de uso único tienden a prevención de daños. Las reglamentaciones varían desde protocolos bien estructurados, como el norteamericano, el australiano y el alemán, a la ausencia de normativa a nivel nacional, como identificado en países desarrollados como Canadá, Japón y algunos países de la Unión Europea. Conclusión: Los controles regulatorios existentes presentan lagunas que dificultan su implementación tanto para los servicios de salud como para los fabricantes. Una metodología alternativa sería la de un sistema regulatorio de productos de uso único centrado en el control de los procesos en lugar de los actuales enfocados en el control del producto.

Palabras clave: Seguridad del paciente. Equipo reutilizado. Política de salud.

'Doutora em Saúde Pública; Professora Adjunta da Universidade do Estado da Bahia (UNEB) - Salvador (BA), Brasil. E-mail: costaeliana2003@hotmail.com Rua Piauí, 269/902 - Pituba - CEP: 41830-270 - Salvador (BA), Brasil.

Recebido: 01 set. 2016 - Aprovado: 27 set. 2016

DOI: 10.5327/Z1414-4425201600040005 


\section{INTRODUÇÃO}

Os produtos médicos são definidos, pelo fabricante, como artigos reusáveis ou de uso único. Os reusáveis ou de uso múltiplo requerem a ação do reprocessamento, processo que consiste em converter um produto contaminado em um dispositivo pronto para uso, incluindo não apenas a limpeza, desinfecção e esterilização do produto, mas também a segurança técnico-funcional por meio de testes de integridade e de funcionalidade. Os produtos de uso único são designados para serem usados somente uma vez, em um único paciente ${ }^{1-6}$.

A prática do reuso de produtos de uso único é uma realidade mundial, iniciada na década de 1970, e desde então, há relatos de reuso desses produtos, mesmo em nações desenvolvidas, e até mesmo naquelas onde o reprocessamento é proibido $^{1-6}$. Essa tendência tem intensificado vários debates e considerações acerca da segurança do paciente, do consentimento informado, das questões econômicas, ambientais, legais e éticas e dos aspectos regulatórios para fabricantes e reprocessadores, denotando interesses distintos por parte dos atores políticos envolvidos: Estado, fabricantes de produtos, serviços de saúde, empresas reprocessadoras, academia, profissionais de saúde, associações de classe e usuários ${ }^{1-12}$.

Dentre os riscos associados ao reuso de produtos médicos, tanto os de uso único quanto os reusáveis, vários autores citam os seguintes ${ }^{1,-7}$ : infecção, biofilmes, contaminação do material com endotoxinas, presença de resíduos tóxicos dos produtos usados para limpeza e desinfecção ou esterilização, bioimcompatibilidade com proteínas dos últimos usuários que eventualmente permaneceram no material, inconfiabilidade funcional, ausência de integridade física e de barreiras de proteção, dentre outros.

No Brasil, o reprocessamento de produtos de uso único é uma realidade nos serviços de saúde e os dados nacionais revelam que essas práticas são comuns em todas as regiões do país, independente do porte e da entidade mantenedora dos hospitais, e que protocolos de reuso são adotados em poucas instituições e na grande maioria de forma inadequada, representando riscos reais para os pacientes usuários desses produtos ${ }^{13-16}$.

Assim, nesse cenário de incremento mundial de produtos médicos nos cuidados de saúde, a regulação do uso e reuso dessas tecnologias tem papel crucial para a implementação de práticas seguras e de prevenção de eventos adversos relacionados a esses produtos. Nesse sentido, este artigo procura responder a seguinte questão central: em que medida os sistemas de regulação sanitária de produtos médicos de uso único adotam políticas dirigidas à prevenção de riscos aos pacientes usuários? Este estudo tem por objetivo analisar o sistema regulatório de reprocessamento de produtos médicos de uso único a nível internacional, incluindo o sistema brasileiro.

\section{MÉTODO}

Trata-se de um estudo de revisão narrativa da literatura, sem restrição de período de tempo de publicação e idioma, realizado por meio de consultas nas bases eletrônicas do Web of Science, Pubmed, Lilacs e SciELO, utilizando os seguintes descritores: reprocessing device medical, reprocessing device single use, reuse device medical, regulation device materials e regulatory devices medicals.

Foram incluídos estudos primários e secundários, selecionados pelo título e resumo. Após leitura dos resumos, foram lidos integralmente apenas os artigos que abordavam aspectos regulatórios dos produtos médicos de uso único, bem como a regulação do reuso e reprocessamento. As referências dos artigos selecionados também foram incorporadas. Os artigos repetidos em mais de uma base de dados foram analisados apenas uma vez. Assim, dos 110 artigos encontrados nas bases eletrônicas supracitadas, foram analisados 33 que atenderam aos critérios de inclusão. Neste estudo, utiliza-se o termo produto médico como sinônimo de produto para saúde, dispositivo, equipamento, material e artigo médico em similaridade com a Agência Nacional de Vigilância Sanitária (ANVISA).

\section{RESULTADOS}

\section{Políticas internacionais de reuso e reprocessamento de produtos médicos de uso único}

O reprocessamento de artigos de uso único é regulamentado e fiscalizado pelo Food and Drug Administration (FDA), que, em 1999-2000, reestruturou sua política de reutilização de dispositivos de uso único, em um princípio de equidade regulatória, no qual fabricantes de produtos originais, empresas reprocessadoras terceirizadas e instituições hospitalares estão em um mesmo patamar de controle regulatório. Ficaram excluídos dessa legislação instituições de saúde não 
hospitalares - clínicas, hospitais-dia, unidades de cuidados prolongados, cuidado domiciliar - , dispositivos de uso único abertos - mas não usados - , marca-passos de implante permanente e hemodializadores ${ }^{3,4,6,9,12}$.

A espinha dessa política regulatória é um esquema de classificação por meio do qual os produtos são categorizados segundo o risco de dano ao paciente baseado na intenção de uso do produto. Há três classes de risco - I, II e III — e dois tipos de submissão de produtos antes da comercialização: a notificação pré-comercialização - $510(\mathrm{~K})$ - e a aplicação de aprovação pré-comercialização — premarket approval application - PMA. O tipo de submissão depende da classificação do produto ${ }^{3,4,6,9,12}$.

O 510 (K), ou notificação pré-comercialização, é a rota mais simples e mais comum para comercialização de um produto médico. Por essa rota, o fabricante deve demonstrar que o novo produto é "substancialmente equivalente" a um produto já comercializado. A assunção é de que o novo produto é tão seguro e efetivo para uso intencionado, desempenha suas funções com tanta consistência quanto um produto já disponível no mercado. O FDA, então, revisa o produto por meio de uma avaliação de similaridade com o dispositivo já comercializado. O PMA é a rota a ser usada se o novo produto não é similar a um já comercializado. Neste caso, o fabricante deve conduzir estudos clínicos para demonstrar segurança e efetividade do produto e o FDA realiza uma inspeção nas instalações dos fabricantes antes da aprovação do PMA. O tempo requerido pelo FDA para a liberação do $510(\mathrm{~K})$ é de aproximadamente 75 a 90 dias e de 180 dias para o $\mathrm{PMA}^{3,4,6,9,12}$.

Atualmente o FDA permite o reprocessamento de mais de cem diferentes produtos de uso único, sendo os cateteres cardiovasculares, fios-guias, circuitos respiratórios, fórceps de biópsia, dispositivos de cauterização, circuitos de aparelhos de anestesia e tubos traqueais os mais reutilizados neste país. Segundo esse órgão, produtos de uso único reprocessados são $50 \%$ mais baratos do que novos ${ }^{3,4,6,9,12}$.

No Canadá, não existe uma regulação única a nível federal e o reprocessamento de produtos de uso único tem sido historicamente delegado para os ministros da saúde das províncias e dos territórios do país. Há relatos de que a reutilização desses produtos ocorre em $40 \%$ das províncias e em $28 \%$ dos hospitais nacionais de cuidado intensivo. Os produtos mais reusados são circuitos ventilatórios e serras. A maioria dos serviços de saúde (85\%) realizam o reprocessamento internamente, mas desde 2014 cresce a tendência do reprocessamento por empresas terceirizadas, a maioria norte-americana licenciada pelo $\mathrm{FDA}^{17-19}$.
Quando o reprocessamento é terceirizado, os hospitais canadenses têm adaptado um sistema de reprocessamento comercial denominado "closed-loop procurement model", por meio do qual o hospital recebe seus próprios produtos médicos encaminhados para reprocessamento para o terceiro setor ou um modelo "open-loop", no qual o hospital não recebe seus próprios produtos de volta, mas compram um "pool" de produtos de uso único reprocessados ${ }^{19}$.

As grandes províncias têm adotado duas posições:

1. proibição do reuso desses produtos, a exemplo de Prince Edward Island, Newfoundland e Labrador e todos três territórios - Northwest, Yukon e Nunavut Alberta, Quebec e New Brunswick; ou

2. permissão do reprocessamento de produtos de uso único apenas por empresas terceirizadas e certificadas por autoridades sanitárias como o Health Canada ou o FDA dos Estados Unidos (EUA) - como ocorre em Bristish Columbia, Manitoba, Ontario, Nova Scotia e Saskatchewan ${ }^{17-19}$.

Na Europa, a União Europeia (UE) não possui uma política única acerca do reprocessamento de produtos de uso único e os Estados-membros têm distintos processos regulatórios $^{19}$. Na Alemanha, desde 2001, a estrutura regulatória existente trata somente dos padrões de qualidade e procedimentos de validação do reprocessamento e torna ilegal a distinção entre uso único e múltiplo dos produtos médicos. O reprocessamento realizado pelo hospital e por empresas terceirizadas é permitido, mas ambos devem implementar sistemas de gerenciamento de qualidade em consonância com a German Act on Medical Devices ${ }^{1,2,11,19}$.

Em outros países da UE, como Reino Unido, Espanha e França, o reprocessamento de artigos descartáveis é proibido por lei desde 2005, mas, efetivamente, o único país que não reutiliza artigos de uso único é a França. Na Espanha, um inquérito realizado em 2005 em 42 hospitais de Madri revelou que $82,4 \%$ deles reprocessavam dispositivos de uso único, sem nenhuma normativa federal que avalie essa prática no país. A Inglaterra permite o reuso de artigos descartáveis somente em situações controladas, devido à grande preocupação com os príons. Na Bélgica, Dinamarca, Holanda, Eslováquia, Suécia e Suiça os produtos médicos de uso único são reprocessados segundo rígidos padrões de qualidade. Na Grécia, na Estônia, no Chipre, na Letônia, em Malta e na Polônia não há regulação sobre essas práticas ${ }^{17,19,20,21}$. 
$\mathrm{Na}$ Ásia, o reuso de produtos descartáveis é comum na maioria dos países e também não há regulações nacionais orientando essas práticas ${ }^{19,20}$. No Japão, o reprocessamento de produtos de uso único não é sistematicamente regulado, e dados apontam que $86,2 \%$ dos hospitais reusam produtos descartáveis e que essas práticas são realizadas de forma inconsistente, sem protocolos e normas estabelecidas ${ }^{20,21}$.

$\mathrm{Na}$ Índia, hospitais rotineiramente reusam produtos de uso único sem regulações conhecidas acerca dessa prática ${ }^{17,19,20}$.

$\mathrm{Na}$ Austrália, o reprocessamento é semelhante às condições dos EUA. Em 2003, o The Australian Therapeutic Goods Administration (TGA), órgão nacional regulador de produtos médicos, introduziu regulações para hospitais e empresas reprocessadoras de produtos de uso único, denominando-os "refabricantes", como descrito na legislação. Devem ajustar-se aos mesmos padrões regulatórios como o fabricante original e são requeridos a demonstrar que os produtos de uso único reprocessados são tão igualmente seguros e que desempenham tão bem quanto um produto novo. A regulação de reprocessamento de produtos de uso único exclui os produtos de uso único abertos, mas não utilizados, e indivíduos que reprocessam dispositivos descartáveis para seu próprio uso pessoal ${ }^{19,20,22,23}$.

Na Nova Zelândia, para reprocessar um produto de uso único, o órgão regulador, Regulator Medsafe, requer aderência com a política regulatória dos EUA ou aprovação com a política australiana ${ }^{20}$.

No Oriente Médio, dados indicam que o reuso desses produtos é comum nos países árabes - particularmente cateteres cardíacos - , a despeito da ausência de uma estrutura regulatória ${ }^{19,20}$.

Israel não possui uma regulação específica para o reprocessamento de produtos de uso único, mas, de modo geral, todo produto médico deve ser registrado no Ministério da Saúde antes de ser comercializado no país. Se o produto é aprovado pelo FDA dos EUA, poderá ser registrado neste país sem nenhum teste adicional. Como em muitos outros países, hospitais de Israel estão reusando muitos produtos de uso único sem qualquer controle federal ${ }^{20}$.

O reino da Arábia Saudita está em processo de implantação de uma política regulatória de produtos médicos. A Saudi Food and Drug Authority emitiu um regulamento provisório em 2008 definindo que um produto médico na Arábia Saudita pode ser comercializado se "aderir aos requerimentos regulatórios aplicados em uma ou mais das jurisdições da Austrália, Canadá, Japão e EUA". Parece que este governo proíbe o reuso de produtos de uso único ${ }^{20}$.

Na África, América Central e América do Sul são prevalentes as práticas de reprocessamento de artigos de uso único por falta de recursos médicos e financeiros ${ }^{19,20}$.

\section{Regulação brasileira de reuso de produtos médicos de uso único}

No Brasil, a ANVISA é o órgão responsável pela regulação do reprocessamento de produtos médicos e, em 2006, editou três normativas ainda em vigor:

1. Resolução da Diretoria Colegiada (RDC) $n^{\circ} 156$, que dispõe sobre o registro, a rotulagem e o reprocessamento de produtos médicos;

2. Resolução Especial (RE) $n^{\circ} 2.605$, que estabelece uma relação de 66 produtos de uso único proibidos de reprocessamento no país; e

3. $\operatorname{RE~}^{\circ} 2.606$, que define as diretrizes para elaboração, validação e implantação de protocolos de reprocessamento de produtos médicos ${ }^{24-26}$.

A Vigilância Sanitária é órgão brasileiro responsável, entre diversas atividades, pela fiscalização do cumprimento das normas que visam proteger a saúde — como a de reprocessamento de produtos médicos.

\section{DISCUSSÃO}

Os EUA, por meio do FDA, possui atualmente o maior controle regulatório estabelecido para as práticas de reuso e reprocessamento de produtos médicos do mundo. Entretanto, as normativas desse órgão apresentam algumas questões que fragilizam esse sistema em pontos cruciais do controle do reprocessamento de produtos, suscitando questões para a implementação dessas normativas, especialmente no que concerne às instituições hospitalares. Inicialmente, o sistema regulatório de produtos médicos do FDA tem como foco norteador da sua política regulatória a comercialização do produto, diferindo da tradicional classificação de risco segundo a possibilidade de infecção envolvida no uso, e artigos considerados críticos - a exemplo de instrumentais cirúrgicos e agulhas - são classificados pelo FDA como classe II (risco médio), e, portanto, com exigências apenas do $510(\mathrm{~K})$ para licenciamento e 
reprocessamento. Por outro lado, o 510 (K) permite que a maioria dos produtos seja comercializado na ausência de altos estudos de qualidade e, portanto, os produtos classe I e a maioria dos classe II recebem a liberação para comercialização na ausência de controles mais acurados de qualidade.

Adicionalmente, a atual política de reprocessamento de produtos de uso único do FDA exige grande capacidade de adaptações para o seu cumprimento, particularmente para os hospitais reprocessadores. As duas submissões para pré-comercialização e/ou reprocessamento de dispositivos médicos - o 510 (K) e o PMA — são ambíguas nas suas exigências para autorização desses processos. Por exemplo, de que maneira o(a) fabricante/reprocessador terceirizado/instituição hospitalar deverá comprovar que o "produto médico reprocessado classe I e II é equivalente em segurança e efetividade a um produto original, não processado", exigido para cumprimento do $510(\mathrm{~K})$ ? O $510(\mathrm{~K})$, com seu controle focado na "equivalência substancial" de um produto já utilizado, permite que a maioria dos produtos nos EUA seja comercializada na ausência de estudos de qualidade mais rigorosos.

Ademais, quais os padrões de controle que as unidades reprocessadoras de materiais usarão para demonstrar "validade científica e evidências clínicas da segurança e efetividade do dispositivo médico de uso único reprocessado da classe III", exigido pelo PMA? Sem metodologia definida, certamente haverá diferentes experiências e ensaios clínicos para o cumprimento dessa normativa. Todas as metodologias apresentadas são aceitas? Outra indefinição refere-se à aceitação pelo FDA de grupos similares de produtos ou se é obrigatório que cada modelo de produto deve submeter-se ao 510 (K) e ao PMA. Por fim, essa regulação isenta outras instituições de saúde que também praticam reuso e reprocessamento de produtos médicos de uso único, a exemplo de clínicas, unidades de cuidado a pacientes crônicos - como psiquiatria -, hospitais-dia e cuidado domiciliar, que permanecem não reguladas. São questões em aberto, que analisamos como lacunas e limitações desse marco regulatório.

No Brasil, os marcos regulatórios vigentes que normatizam o reprocessamento de produtos constituem avanços na normatização do reprocessamento de produtos médicos no nosso país. Entretanto, existem várias imprecisões e conteúdo abstrato nessas legislações, que propiciam diversificadas interpretações e problematizam sua operacionalização por parte dos serviços de saúde, das empresas terceirizadas de reprocessamento e dos fabricantes ou importadores desses produtos.

A Resolução 156/ 2006 categoriza os produtos médicos como "passíveis de reprocessamento" e de "reprocessamento proibido" e estabelece que esse enquadramento deve ser feito no registro do produto, quando o fabricante ou importador deve apresentar à ANVISA documentação que fundamente a indicação. No entanto, essa normativa não especifica a documentação requerida e os parâmetros de avaliação para fabricantes ou importadores, no ato de inscrição e registro de produtos permanentes e de uso único. A grande pergunta é: qual o critério que essa Agência utiliza para aceitar ou rejeitar a classificação do produto referida pelos fabricantes no momento do registro? Quais testes são requeridos dos fabricantes pela ANVISA para comprovar que o produto é reusável ou de uso único no ato do registro?

A RE $2.605 / 2006$ lista 66 produtos enquadrados como de uso único e proibidos de reprocessamento, mas não explicita os critérios utilizados na escolha dos artigos que compõem essa lista negativa. Tal formulação não favorece a compreensão das bases técnico-científicas da regulamentação de uma prática que envolve questões tão relevantes para a saúde no país. Os questionamentos são vários: por que alguns produtos passíveis de reuso, como sugadores e diques de borracha odontológicos, luvas e compressas compõem a lista negativa de reuso e outros comprovadamente de alto risco, como pinças de biópsia endoscópica, papilótomos, kits de vitrectomia e tantos outros do cuidado assistencial também de alto risco não foram incluídos? Como lidar com a incorporação crescente desse arsenal tecnológico com uma lista finita de produtos? Por que a opção de trabalhar com uma lista passível de tornar-se obsoleta, como já está, e centrar o foco no produto em lugar dos processos que envolvem as etapas do reprocessamento?

A RE 2.606/2006 define que as empresas terceirizadas e os serviços de saúde que reprocessam artigos críticos e semicríticos devem elaborar, validar e implantar protocolos para cada marca e tipo de produto selecionado, com descrição detalhada de todos os passos do reprocessamento, além da garantia da qualidade do resultado de todas as fases, incluindo a avaliação de funcionalidade, esterilidade, rastreabilidade, condições de armazenamento e descarte de cada produto reprocessado.

Normatiza, também, que cada produto crítico e semicrítico a ser reprocessado, sem especificar se é de uso 
único ou múltiplo, deve ter um prontuário com informações referentes ao artigo - dimensões, estrutura, composição - , registro na ANVISA, fabricante e fornecedor, nome do responsável pelo reprocessamento e local e data de cada reprocessamento. Essa normativa, ao tempo que exige a elaboração, validação e implantação de protocolos de reprocessamento de produtos médicos, não indica qual a metodologia aceitável para a validação dos processos a serem realizados pelos hospitais, o que não apenas dificulta a busca por sua operacionalização, mas também dá margem à realização de protocolos de validação com resultados duvidosos, trazendo insegurança no reprocessamento de produtos.

Ademais, essa regulamentação é imprecisa quando exige garantia de qualidade de todas as etapas do processo, incluindo avaliação da funcionalidade, esterilidade, pirogenicidade, atoxicidade e integridade. Novamente, interroga-se qual a metodologia aceitável para esses controles de qualidade. Há que se fazer esses testes para todos os artigos críticos e semicríticos? Como, quem e quando avaliar funcionalidade e integridade de todos os artigos reprocessados, tendo em conta a imensidão de produtos médicos existentes em uma instituição de saúde? Qual deverá ser a periodicidade mínima desses testes? Como elaborar um prontuário para cada artigo crítico e semicrítico com os dados exigidos por essa normativa, considerando a realidade físico-funcional-organizacional da maioria dos Centros de Material e Esterilização (CMEs) dos hospitais do Brasil e a vastidão de produtos que compõem o seu arsenal? São questões em aberto desse marco regulatório que impactam negativamente na operacionalização dos serviços de saúde.

Além das questões suprarreferidas, como a Vigilância Sanitária pode monitorar/fiscalizar/controlar essa normativa nas inúmeras unidades de saúde do país? Os profissionais de vigilância sanitária possuem a expertise necessária para realizar o controle sanitário do reuso de produtos médicos? Essas são outras questões dessa temática em aberto no Brasil.

Sem a devida fiscalização, estudos comprovam que a regulamentação do reprocessamento de produtos médicos, editada desde 2006, vem sendo retardada ou boicotada em sua implementação nos hospitais brasileiros ${ }^{13-16}$. Além das questões que suscita, tal retardo desafia a própria legitimidade da norma, reafirmando a problemática que envolve a reutilização de produtos médicos, tanto os reusáveis quanto os considerados de uso único.

\section{CONCLUSÃO}

A revisão da literatura registra que internacionalmente há uma variedade de níveis regulatórios no estabelecimento de políticas de reuso de produtos médicos de uso único, que, de modo geral, tendem a ter um caráter preventivo, com recomendações que objetivam a segurança da saúde pública.

Embora essas regulamentações tenham diferenças substanciais, o princípio do gerenciamento de risco deveria ser seu elemento norteador e o grau de escrutínio regulatório imposto para qualquer produto médico, independente se de uso único ou múltiplo, deveria ser proporcional à intenção de uso do dispositivo, ao seu nível de risco e ao grau de invasividade do produto no corpo humano.

As regulamentações variam desde protocolos bem estruturados, como o norte-americano, o australiano e o alemão, à ausência de normatização a nível nacional, também identificada em países desenvolvidos como Canadá, Japão e alguns países da União Europeia, na Ásia e no Oriente Médio, apontando para a falta de prioridade política para a questão da problemática que envolve o reuso de produtos médicos.

Mesmo existentes, os controles regulatórios dos países, como os dos EUA, da Austrália e do Brasil, apresentam grandes lacunas, como as já apontadas neste estudo, dificultando sua implementação tanto para os serviços de saúde quanto para os fabricantes.

No Brasil, a fiscalização da implementação dessas normativas pela Vigilância Sanitária também é outra questão em aberto quanto à verdadeira capacidade técnico-operacional desse órgão para o efetivo controle sanitário do reuso de produtos médicos no país.

Talvez uma metodologia alternativa seja a de desenvolver um sistema regulatório de reuso e reprocessamento de produtos de uso único centrado no controle dos processos em lugar dos atuais, focados no controle dos produtos mundialmente implantados. 


\section{REFERÊNCIAS}

1. Kraft M. Framework conditions and requeriments to ensure the technical functional safety of reprocessed medical devices. GMS Krankenhhyg Interdiszip. 2008;3(3):Doc23.

2. Grobkopf $\mathrm{V}$, Jakel $\mathrm{C}$. Legal framework conditions for the reprocessing of medical devices. GMS Krankenhaushyg Interdiszip. 2008;3(3):Doc24.

3. Department of Health and Human Services. Food and Drug Adminstration (FDA). Center for Devices and Radiological Health (CDRH). Reprocessing and reuse of single-use devices: Review Priorization Scheme. Rockville; 2000.

4. United States General Accounting Office. Health Education and Human Services Division. Single-Use Medical Devices. Little Available Evidence of Harm From Reuse, but Oversigth Warrented. Report to Congressional Requesters. Washington, D.C.; 2000.

5. Health Canada. Therapeutic Products Directorate Holland Cross. Reprocessing of reusable and Single-use Medical Device. Tower B. Ottawa, Ontario; 2004.

6. Day P. What is the evidence on the safety and effectiveness of the reuse of medical devices labelled as single-use only? New Zealand Health Tecnology Assessment (NZHTA). NZHTA Tech Brief Series. 2004;3(2). [acessado 2015 maio 2]. Disponível em: http://www. enfermeriajw.cl/pdf/normas/REUSE\%20MEDICAL\%20DEVICES.pdf

7. Altenstetter C. EU and Member State Medical Devices Regulation. Int J Technol Assess Health Care. 2003;19(1):228-48.

8. Peláez B, Andrade R, Díaz P, Cano S, Barriuso E, Fereres J. Reutilización de dispositivos médicos de um solo uso. Serviço de Medicina Preventiva. Hospital Clínico San Carlos. Madri; 2009.

9. Reed SD, Shea AM, Schulman KA. Economic implications of potential changes to regulatory and reimbursement policies for medical devices. J Gen Intern Med. 2008;23(Suppl 1):50-6.

10. Feldman MD, Petersen AJ, Karliner LS, Tice JA. Who is responsible for evaluating the safety and effectiveness of medical devices? The role of independent technology assessment. J Gen Intern Med. 2008;23(Suppl 1):57-63.

11. Lamph S. Regulation of medical devices outside the European Union. J R Soc Med. 2012;105(Suppl 1):S12-21.

12. Hakansson MA. Reuse versus single-use catheters for intermittent catheterization: what is safe and preferred? Review of current status. Spinal Cord. 2014;52(7):511-6.

13. Oliveira AC, Oliveira KA, Noronha AHT, Gomes OMS, Braga FB. Reprocessamento de produtos de uso único nas instituições hospitalares de Belo Horizonte. REME Rev Min Enf. 2006;10(2):138-44.
14. Amarante JBM, Toscano CM, Pearson ML, Roth V, Jarvis WR, Levin AS. Reprocessing and reuse of single-use medical devices used during hemodynamic procedures in Brazil: a widespread and largely overlooked problem. Infect Control Hosp Epidemiol. 2008;29(9):854-8.

15. Bonfim FMTS, Lima SG, Victor EG. Análise do reprocessamento de cateteres de hemodinâmica em uma capital brasileira. Rev Bras Cardiol. 2013;26(1):33-9.

16. Costa EAM, Costa EA. Risco e segurança sanitária: análise do reprocessamento de produtos médicos em hospitais de Salvador, BA. Rev Saúde Pública. 2012;46(5):800-7.

17. Hussain M, Balsara KP, Nagral S. Reuse of single-use devices: looking back, looking forward. Natl Med J India. 2012;25(3):151-55.

18. Shuman EK, Chenoweth CE. Reuse of medical devices: implication for infection control. Infect Dis Clin North Am 2012;26(1):165-72.

19. Health Canada. Canadian Agency for Drugs and Technologies in Health (CADTH). Reprocessing of single-use medical devices: a 2015 Update. Issue 48. Ontario; 2015.

20. Association of Medical Device Reprocessors (AMDR). AMDR Summary: International Regulation of single use medical device reprocessing.

21. Collier R. Reprocessing single-use devices: an international perspective. CMAJ [Internet]. 2011;183(11):1244. [acessado 2015 fev 2]. Disponível em: http://www.ncbi.nlm.nih.gov/pmc/articles/PMC3153511

22. Kok A, Kawahara K. Current practices and problems in the reuse of single-use devices in Japan. J Med Dent Sci. 2005;52(1):81-9.

23. Medicine and Healthcare Products Regulatory Agency. Regulating Medicines and Medical Devices (MHRA). Single-use medical devices: implications and consequences of reuse. MHRA; 2013. [acessado 2015 fev 2]. Disponível em: www.gov.uk/government/publications/ single-use-medical-devices-implications-and-consequences-of-re-use

24. Agência Nacional de Vigilância Sanitária (ANVISA). Resolução RDC №. 156, de 11 de agosto de 2006. Dispõe sobre registro, rotulagem e reprocessamento de produtos médicos e dá outras providências. Brasília: Diário Oficial da União; 15 fev. 2006.

25. Agência Nacional de Vigilância Sanitária (ANVISA). Resolução - RE Nº 2.605, de 11 de agosto de 2006. Contém a lista de produtos que não podem ser reprocessados. Brasília: Diário Oficial da União; 15 fev. 2006.

26. Agência Nacional de Vigilância Sanitária (ANVISA). Resolução - RE $N^{\circ} 2.606$, de 11 de agosto de 2006. Dispõe sobre as diretrizes para elaboração, validação e implantação de protocolos de reprocessamento de produtos médicos e dá outras providências. Brasília: Diário Oficial da União; 15 fev. 2006. 